\title{
New concepts of coagulation and bleeding in liver disease
}

\author{
Patrick G. Northup · Stephen H. Caldwell
}

Received: 16 December 2009/Accepted: 21 December 2009/Published online: 16 January 2010 (C) SIMI 2010

The bleeding tendencies of patients with chronic liver disease have challenged clinicians for centuries. In recent decades, modern science has helped in elucidating some of the mechanisms for this coagulopathy, but there is much to be discovered. It is well known that progressive liver disease is associated with increasing disruption in protein synthesis. Indeed, one of the most ubiquitous of serum proteins, albumin, has been intricately linked to prognosis in cirrhosis patients through the Child-Turcotte-Pugh Score [1], and clinicians have known that dwindling albumin levels are predictive of poor prognosis in these patients. However, it is less widely understood that patients with chronic liver disease may have a persistence of some serum proteins due to ineffective protein degradation. This combination of protein deficiency and persistence contributes to the conflicting knowledge base on coagulopathy in liver disease. In the healthy state of hemostasis, the bleeding and clotting tendencies are balanced. In cirrhosis, this balance is clearly achieved most of the time. The key concept is that the balance is tenuous because of decreased reserve, and when disrupted there can be faltering towards bleeding, especially in the cases where mechanical issues are involved, such as procedural or variceal bleeding. However, there are also circumstances that can lead to abnormal clotting in these patients because of flow issues and local persistence of the prothrombotic proteins. It is the understanding and quantification of this tenuous balance

P. G. Northup $(\varangle) \cdot$ S. H. Caldwell

Division of Gastroenterology and Hepatology,

University of Virginia, JPA and Lee Street,

MSB 2142, P.O. Box 800708, Charlottesville,

VA 22908-0708, USA

e-mail:pgn5qs@virginia.edu between bleeding and clotting that is shaping our current understanding of the coagulopathy of liver disease.

The quantification of this imbalance is the key to using this conceptual framework to establish a clinically useful paradigm for treating patients with chronic liver disease. The widely available clinical laboratory tests used to measure coagulopathy were generally developed to measure quantitative deficiencies in platelet counts or procoagulant factors, and were designed to assess congenital factor deficiencies, such as hemophilia and acquired platelet diseases, such as idiopathic thrombocytopenic purpura. Indeed, the international normalized ratio (INR) was first conceived as a means of standardizing treatment with warfarins. Recent commentary by Tripodi et al. [2], in this journal has outlined the difficulty in using traditional laboratory testing to measure the three fundamental coagulation phases in patients with cirrhosis.

In primary hemostasis, which fundamentally involves the platelets and tissue factor, there is a near universal quantitative decrease in platelet numbers in cirrhosis patients, but the data supporting a primary platelet functional deficit is less strong. Based on the level of absolute thrombocytopenia commonly seen in advanced liver disease and early studies suggestive of an additional platelet function disorder [3], it was thought that primary hemostasis was markedly reduced in these patients. However, because much of the function of platelets is dependent on the flow conditions within the vessels, in vitro tests are not representative of the true in vivo function of the platelet. Lisman et al. [4], have extensively studied platelets in flow conditions, and find adequate platelet function using serum of cirrhosis patients due to elevated antigen levels of von Willebrand factor and reduced activity of its cleaving protein, ADAMTS13 [5]. This research has brought into question the magnitude of defect in primary hemostasis in 
chronic liver disease. Even the oldest in vivo test of platelet function, the bleeding time, has failed to predict clinical gastrointestinal bleeding [6] or bleeding after major hepatectomy [7] and has been largely abandoned as a routinely used test. It is clear that a minimum number of platelets are needed to generate enough thrombin burst to initiate adequate hemostasis. Although highly variable from patient to patient, it appears that a platelet count above 50,000 per $\mathrm{m}^{3}$ is likely to be adequate based on endogenous thrombin potential studies [8].

Clot formation is the second phase of hemostasis that is disrupted in chronic liver disease. It is well known that a quantitative deficit in standard procoagulant proteins is easily measured using traditional laboratory assessments, such as the activated partial thromboplastin time (aPTT) and the prothrombin time (PT). However, these traditional assays do not represent the in vivo situation with regards to available levels of other critical components of the clotting system, including tissue factor and the innate anticoagulants protein $\mathrm{C}$, protein $\mathrm{S}$, and antithrombin. Recently, Tripodi et al. [9] report that when measured in the presence of adequate thrombomodulin, which is the main activator of the protein $\mathrm{C}$ system of clot degradation, serum from cirrhosis patients has a relative deficiency of protein $\mathrm{C}$ activity. The level of protein $\mathrm{C}$ deficiency measured in this study is comparable to the level in patients with congenital protein $\mathrm{C}$ deficiency. This finding once again underscores the lack of predictability of standard laboratory testing of coagulopathy when used in the cirrhosis patients.

Finally, there is no good measure that is clinically available for the final phase of coagulation: fibrinolysis. Using thromboelastography and euglobulin lysis times, the estimated prevalence of hyperfibrinolysis in hospitalized cirrhosis patients is about $31 \%[10,11]$ although the clinical syndrome of persistent mucosal and puncture wound bleeding is much less common in practice. This clinical syndrome is likely due to abnormal local persistence of thrombin-activatable fibrinolysis inhibitor (TAFI), causing early clot breakdown and instability of hemostasis due to premature clot dissolution. This syndrome does clinically respond to the plasminogen inhibitor epsilon-aminocaproic acid [12], but clinically available testing for this disorder is lacking, and misdiagnosis and inappropriate use of this agent could be problematic. This clinical syndrome is yet another example of how outdated clinical laboratory testing available to the practitioner severely limits the expansion of therapeutic options in the area of coagulopathy in liver disease.

Despite the well-known risks for massive bleeding related to gastroesophageal varices and invasive procedures, there is mounting evidence that a tendency towards clotting may impact a significant portion of stable cirrhosis patients. This topic has been covered extensively elsewhere
[13], but the adverse effects of macrovascular clots, such as portal vein thrombosis and hepatic vein thrombosis are undeniable. In addition, there is mounting single-center [14] and population-based [15] evidence that peripheral thromboembolic phenomena (deep vein thrombosis and pulmonary embolism) are not rare in cirrhosis patients, and may be overlooked without thinking of their possibility and initiating an aggressive search. The macrovascular clots are an acute problem for the clinician and patient, but a longterm concern for chronic liver disease patients with hypercoagulation relates to progression of disease and fibrosis due to the possibility of parenchymal extinction [16], progression of hepatitis $\mathrm{C}$ infection [17], and the development of fibrosis in non-alcoholic fatty liver disease [18].

The paradox with the above arguments is clear to the practicing physician. The in vitro laboratory testing suggests that stable patients with chronic liver disease are in a tenuous balance of coagulation with some tendency toward clotting; yet, the most profound clinical events in the course of liver disease tend to be involved with hemorrhage. One of the fundamental reasons for this seeming contradiction is related to the lack of tests that are reflective of the local conditions at the site of tissue injury. The modern understanding of the coagulation cascade is fundamentally based on the exposure of tissue factor to the local vascular environment, the production of a thrombin burst, localized clotting and eventually fibrinolysis resulting in localized hemostasis. Because of the difficulty in studying local conditions at the site of injury, not much is known about how these factors influence the macroscopic bleeding or clotting risk. Recent investigation in the areas of coagulation in flow conditions [5], nitric oxide and endothelial function related to liver disease [19], endogenous heparinoids and their role in coagulation [20], and tissue factor behavior at the local levels [21] show promise in elucidating better clinical laboratory testing for systemic coagulation risk in patients with chronic liver disease. Until reliable tests of local clotting activity are available, researchers in coagulation must develop clinically useful tests to assess the tendency of an individual patient to have bleeding or clotting complications. These tests might take the form of a general "clotting profile" for each individual patient that may incorporate elements of clotting factor reserve, platelet function, endothelial function, and fibrinolytic activity. Development of a clotting profile is of utmost importance in patient care, but is also of paramount interest for the use in the clinical trial setting.

The lack of clinical trials of coagulopathy in liver disease is largely due to the inability to develop satisfactory surrogate endpoints in measuring coagulation. One common clinical question is the use of prophylactic freshfrozen plasma (FFP) prior to invasive procedures. It has been shown in observational studies that the use of FFP in 
cirrhosis patients is a major component of the blood product usage in a hospital, and much of it is used in prophylaxis prior to procedures [22]. A case study in the failure of a clinical trial due to the lack of a surrogate coagulopathy endpoint was the fresh-frozen plasma infusions to reduce risk of bleeding related to invasive procedures (SHIP) trial sponsored by the US National Institutes of Health [23]. This trial was a multicenter, randomized controlled trial designed to assess the ability of FFP to prevent bleeding in subjects undergoing invasive biliary procedures or liver biopsy. The target population was defined as having "moderate coagulation defect" using a platelet count $>70,000$ per $\mu$ l, INR between 1.3 and 1.9 , and aPTT $<50 \mathrm{~s}$. The targeted enrollment was a total 1,300 subjects. After more than 13 months, the study was suspended and eventually terminated because of inadequate enrollment. It was speculated that the physicians performing procedures in the study centers would not allow their patients to enroll in a study that involved no plasma transfusion prior to the procedure. This expensive clinical trial failure might have been avoided with adequate coagulation profiles in the participants showing the individual tendency for bleeding or clotting. A proper endpoint in future studies such as this will need to be a change in a validated coagulation profile, not clinical bleeding. This example underscores the urgent need for appropriate laboratory testing that will be the required first step in evaluating the multiple therapeutic and prophylactic options that are on the horizon.

In summary, the complex nature of local versus systemic coagulopathy in cirrhosis and the multitude of cofactors influencing the coagulation system in health and in disease states make the routine assessment of bleeding or clotting risk in the patient with chronic liver disease challenging. Recent work by many talented investigators has opened the door in promising new clinical laboratory testing paradigms that have promise for future breakthroughs. However, our current testing methods are outdated, not validated or designed for use in patients with liver disease, are inadequate to base clinical decisions at the patient level, and are unacceptable for use as endpoints in clinical therapeutic trials. The primary research focus should immediately be aimed at defining a coagulation profile for individual patients that better defines the tenuous balance between bleeding and clotting. After this profile has been validated in the research setting, welldesigned randomized clinical trials are needed to totally reassess our understanding and use of both prophylactic and therapeutic interventions on the patient with chronic liver disease. It is only with this reassessment of our current research and clinical environments that we can push the knowledge of coagulopathy in liver disease into the future.
Conflict of interest None.

\section{References}

1. Christensen E, Schlichting P, Fauerholdt L, Gluud C, Andersen PK, Juhl E, Poulsen H, Tygstrup N (1984) Prognostic value of Child-Turcotte criteria in medically treated cirrhosis. Hepatology 4(3):430-435

2. Tripodi A, Primignani M, Mannucci PM (2009) Abnormalities of hemostasis and bleeding in chronic liver disease: the paradigm is challenged. Intern Emerg Med. doi:10.1007/s11739-009-0302-z

3. Younger HM, Hadoke PW, Dillon JF, Hayes PC (1997) Platelet function in cirrhosis and the role of humoral factors. Eur J Gastroenterol Hepatol 9(10):989-992

4. Lisman T, Adelmeijer J, de Groot PG, Janssen HL, Leebeek FW (2006) No evidence for an intrinsic platelet defect in patients with liver cirrhosis-studies under flow conditions. J Thromb Haemost 4(9):2070-2072

5. Lisman T, Bongers TN, Adelmeijer J, Janssen HL, de Maat MP, de Groot PG, Leebeek FW (2006) Elevated levels of von Willebrand Factor in cirrhosis support platelet adhesion despite reduced functional capacity. Hepatology 44(1):53-61

6. Basili S, Ferro D, Leo R, Juliano L, Alessandri C, Cordova C, Violi F (1996) Bleeding time does not predict gastrointestinal bleeding in patients with cirrhosis The CALC Group. Coagulation abnormalities in liver cirrhosis. J Hepatol 24(5):574-580

7. Wong AY, Irwin MG, Hui TW, Fung SK, Fan ST, Ma ES (2003) Desmopressin does not decrease blood loss and transfusion requirements in patients undergoing hepatectomy. Can J Anaesth 50(1):14-20

8. Tripodi A, Primignani M, Chantarangkul V, Clerici M, Dell'Era A, Fabris F, Salerno F, Mannucci PM (2006) Thrombin generation in patients with cirrhosis: the role of platelets. Hepatology 44(2):440-445

9. Tripodi A, Primignani M, Chantarangkul V, Dell'era A, Clerici M, de Franchis R, Colombo M, Mannucci PM (2009) An imbalance of pro- vs anti-coagulation factors in plasma from patients with cirrhosis. Gastroenterology 137(6):2105-2111

10. Hu KQ, Yu AS, Tiyyagura L, Redeker AG, Reynolds TB (2001) Hyperfibrinolytic activity in hospitalized cirrhotic patients in a referral liver unit. Am J Gastroenterol 96(5):1581-1586

11. Violi F, Ferro D, Basili S, Quintarelli C, Musca A, Cordova C, Balsano F (1993) Hyperfibrinolysis resulting from clotting activation in patients with different degrees of cirrhosis. The CALC Group. Coagulation abnormalities in liver cirrhosis. Hepatology 17(1):78-83

12. Gunawan B, Runyon B (2006) The efficacy and safety of epsilonaminocaproic acid treatment in patients with cirrhosis and hyperfibrinolysis. Aliment Pharmacol Ther 23(1):115-120

13. Northup PG, Sundaram V, Fallon MB, Reddy KR, Balogun RA, Sanyal AJ, Anstee QM, Hoffman MR, Ikura Y, Caldwell SH (2008) Hypercoagulation and thrombophilia in liver disease. J Thromb Haemost 6(1):2-9

14. Northup PG, McMahon MM, Ruhl AP, Altschuler SE, VolkBednarz A, Caldwell SH, Berg CL (2006) Coagulopathy does not fully protect hospitalized cirrhosis patients from peripheral venous thromboembolism. Am J Gastroenterol 101(7):15241528; quiz 1680

15. Sogaard KK, Horvath-Puho E, Gronbaek H, Jepsen P, Vilstrup H, Sorensen HT (2009) Risk of venous thromboembolism in patients with liver disease: a nationwide population-based case-control study. Am J Gastroenterol 104(1):96-101

16. Wanless IR, Wong F, Blendis LM, Greig P, Heathcote EJ, Levy G (1995) Hepatic and portal vein thrombosis in cirrhosis: possible 
role in development of parenchymal extinction and portal hypertension. Hepatology 21(5):1238-1247

17. Poujol-Robert A, Rosmorduc O, Serfaty L, Coulet F, Poupon R, Robert A (2004) Genetic and acquired thrombotic factors in chronic hepatitis C. Am J Gastroenterol 99(3):527-531

18. Wanless IR, Shiota K (2004) The pathogenesis of nonalcoholic steatohepatitis and other fatty liver diseases: a four-step model including the role of lipid release and hepatic venular obstruction in the progression to cirrhosis. Semin Liver Dis 24(1):99-106

19. Annie-Jeyachristy S, Geetha A, Surendran R (2008) Changes in the level of cytosolic calcium, nitric oxide and nitric oxide synthase activity during platelet aggregation: an in vitro study in platelets from normal subjects and those with cirrhosis. J Biosci 33(1):45-53
20. Smalberg JH, Leebeek FW (2009) Superimposed coagulopathic conditions in cirrhosis: infection and endogenous heparinoids, renal failure, and endothelial dysfunction. Clin Liver Dis 13(1):33-42

21. Panasiuk A, Zak J, Panasiuk B, Prokopowicz D (2007) Increase in expression of monocytic tissue factor (CD142) with monocytes and blood platelet activation in liver cirrhosis. Blood Coagul Fibrinolysis 18(8):739-744

22. Shah N, Xavier E, Caldwell SH (2009) Procoagulant blood product usage in liver disease patients. J Hepatol 50(supp 1):S185

23. National Heart, Lung, and Blood Institute (NHLBI) Fresh-frozen plasma infusions to reduce risk of bleeding related to invasive procedures. http://clinicaltrials.gov/ct2/show/NCT00233246 